\title{
Seismic Performance Evaluation of Improved Jet Grouted Grid Form with Horizontal Slab in Liquefaction Mitigation
}

\author{
Myat Myat Phyo Phyo', Hemanta Hazarika², Li Shi', Hiroaki Kaneko³, Tadashi Akagawa3 \\ ${ }^{1}$ Department of Civil and Structural Engineering, Kyushu University, Fukuoka, Japan \\ ${ }^{2}$ Department of Civil Engineering, Kyushu University, Fukuoka, Japan \\ ${ }^{3}$ Japan Foundation Engineering Co., Ltd., Tokyo, Japan \\ Email: phyo.myat.692@s.kyushu-u.ac.jp, hazarika@civil.kyushu-u.ac.jp, li.shi.229@s.kyushu-u.ac.jp, \\ hiroaki_kaneko@jafec.co.jp, tadashi_akagawa@jafec.co.jp
}

How to cite this paper: Phyo, M.M.P., Hazarika, H., Shi, L., Kaneko, H. and Akagawa, T. (2020) Seismic Performance Evaluation of Improved Jet Grouted Grid Form with Horizontal Slab in Liquefaction Mitigation. Open Journal of Civil Engineering, 10, 301-320.

https://doi.org/10.4236/ojce.2020.103024

Received: June 29, 2020

Accepted: September 26, 2020

Published: September 29, 2020

Copyright ( 2020 by author(s) and Scientific Research Publishing Inc. This work is licensed under the Creative Commons Attribution International License (CC BY 4.0).

http://creativecommons.org/licenses/by/4.0/

\begin{abstract}
A chain event of the 2016 Kumamoto earthquakes caused considerable geotechnical damage related to liquefaction in many places around Kumamoto plain. Many low-rise houses and traditional Japanese style houses, which were constructed on shallow foundation, suffered differential settlement and tilting due to liquefaction. To mitigate the building damages due to the liquefaction, a new countermeasure method of jet grout grid form with a horizontal slab is introduced in this study. The effectiveness of the proposed technique was evaluated through physical modelling and numerical modelling. As a part of the physical modelling, a set of $1 \mathrm{~g}$ shaking table tests for unimproved case and improved case were performed, in which the mitigation effects of the grid form with a horizontal reinforcing slab were examined based on the acceleration, excess pore water pressure ratio as well as ground settlement. Numerical simulation was also performed for assessing the effect of improved method on soil-structure interaction and building settlement during the earthquake. The physical and numerical results confirmed that the grid form with horizontal slab reinforced method is effective in settlement control and offers favorable contribution in liquefaction mitigation.
\end{abstract}

\section{Keywords}

Liquefaction Mitigation, Jet Grouting, Shaking Table Tests, Numerical Simulation

\section{Introduction}

The 2016 Kumamoto Earthquakes are a series of strong earthquakes including a 
6.5 magnitude foreshock and a 7.0 magnitude main shock, both struck at $\mathrm{Ku}$ mamoto City, Kumamoto Prefecture on Kyushu Island, Japan. Subsequently, a chain of earthquakes triggered significant damage to infrastructures including the renowned cultural heritage of Kumamoto castle, buildings, roads and river embankments. In addition, there were geotechnical damages mostly related to liquefaction and associated lateral spreading in a few districts of Kumamoto City and in the port areas [1]. Especially, the Mashikitown and Minami ward of Kumamoto City experienced liquefaction extensively and intensively. Several traditional Japanese style houses and low-rise buildings suffered differential settlement and tilting as the consequences of liquefaction in those areas. Figure 1(a) indicates the building damage due to differential settlement observed in a residence in Makishi town, Kumamoto City. Furthermore, a new private hospital in Hirata district of Minami ward was one of the most affected buildings due to liquefaction. The ground subsidence about $40 \mathrm{~cm}$ was observed around the building as shown in Figure 1(b). According to the Kumamoto City report, approximately 2900 buildings suffered severe damage caused by liquefaction during the 2016 Kumamoto earthquake [2].

In view of the aforesaid statements, liquefaction can cause substantial damage to buildings in the form of ground subsidence and bearing capacity failure of the soil. In order to mitigate the damage caused by such future liquefaction, it is necessary to take appropriate remediation measures in geotechnical engineering practice. Several countermeasure methods have been developed to mitigate liquefaction and strengthen ground, such as gravel drain method, sand compaction pile method, cement deep mixing method, etc. Among many ground improvement techniques, a grid shaped cement mixing method is frequently used in Japan and its high applicability effect has been evaluated in many earthquakes [3] [4]. Many centrifuge models tests, large scale shaking table tests and numerical analyses were performed to assess the effectiveness of grid form method in liquefaction mitigation, proposing the design procedures and guidelines [5] [6] [7]. However, the number of researches for grid form method in liquefaction mitigation is very limited and some issues still remain in facilitating conventional grid form countermeasure method in residential areas. Firstly, the

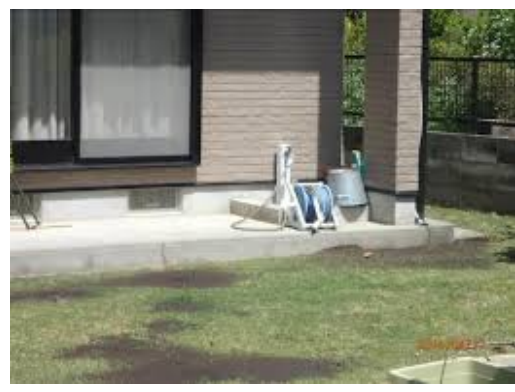

(a)

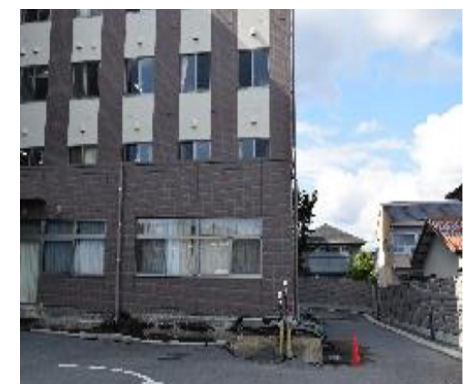

(b)

Figure 1. Buildings damage due to differential settlement in Kumamoto City (a) Mashiki town; (b) Hospital building in Minami wards [1]. 
construction cost is high, and it may not be economical to install under normal detached houses. Secondly, the equipment used in conventional method requires space and is difficult to utilize in residential areas. Thirdly, the construction of grid form directly under existing houses is not feasible, thereby grid spacing ratio is wide to avoid the house and it leads to increase the liquefaction potential. To overcome these problems, the present study is aimed at proposing a new grid from liquefaction countermeasure method with more economical and effective construction approach. The new countermeasure method of jet grout columns with horizontal slab introduced in this study offers low cost construction approach and fulfils the space restraint requirement. In addition, it is applicable to apply near boundaries of existing structures. Due to enforcement with the horizontal slab, it also enables to strengthen the soil and provide support to the structure similarly as the large diameter jet grout wall used in conventional method, despite having thinner walls $(0.15 \mathrm{~m})$. Moreover, the use of small grid spacing ratio (0.2) in this method helps to suppress the generation of excess pore water pressure.

This present study focuses on evaluating the effectiveness of proposed grid form countermeasure method with the horizontal slab in reducing liquefaction risk. In this paper, the previous researches and development of the new countermeasure method are introduced first. Subsequently in the later sections, the experimental modelling and numerical simulation performed were described in alignment with their corresponding results. For experimental modelling, $1 \mathrm{~g}$ shaking table tests for unimproved case and improved case were conducted under different dynamic loading conditions. The salient results of the acceleration response, excess pore water pressure ratio and settlement observed from both cases were discussed to assess the effectiveness of the soil improvement method. In numerical case, an effective stress-based model UBC3D-PLM was employed in liquefiable sand and the parameters used in this model were calibrated prior to the analyses to confirm the reliability of the numerical results. Herein, for both unimproved and improved ground, the focus is given to the significance of the settlement and distribution of shear strain under the building evaluated by using the numerical PLAXIS software.

\section{New Countermeasure Method for Liquefaction Mitigation}

One widely used existing method against liquefaction is grid type soil reinforcing method. In this method, the stiff grid walls are made of overlapped independent columns constructed using soil cement mixing method or jet-grouting method. It is expected to restrict shear deformation of the liquefiable soil within the grid form, so that excess pore water pressure generation can be suppressed. This mechanism of the grid from improved method is presented in Figure 2. In fact, the grid form method was developed since after early 1990s, but its effectiveness was first evaluated in the Hyogoken, Nanbu earthquake in 1995 [3]. Many numerical analyses, physical model tests and field tests were performed to 


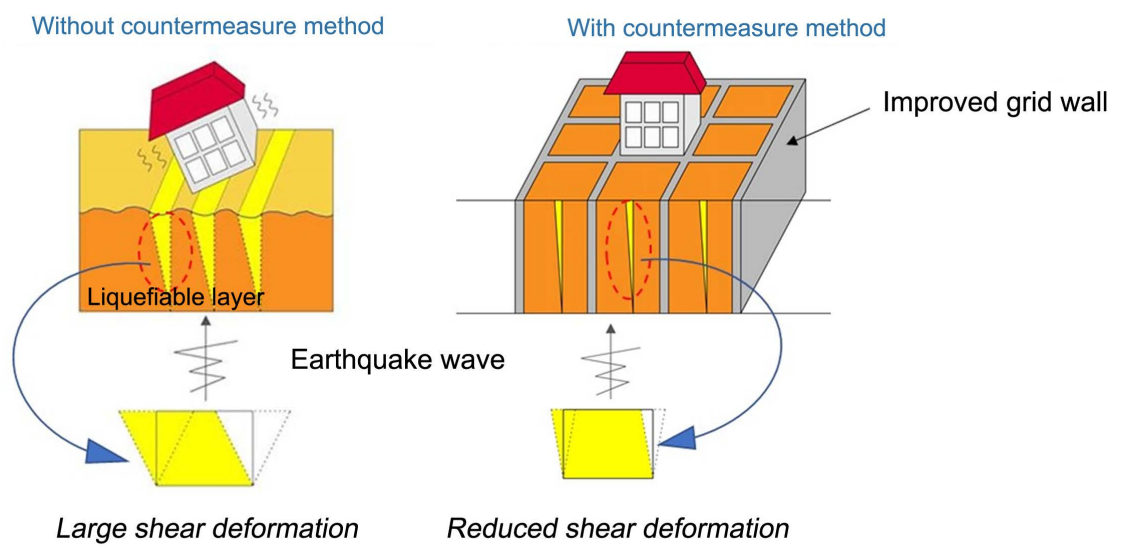

(a)

(b)

Figure 2. Liquefaction mitigation mechanism of grid form wall (a) Earthquake induced large shear deformation occurred in unimproved case (b) Reduced shear deformation due to reinforcing effect of the improved grid form method.

investigate the performance of the grid spacing, interaction between improved ground and surrounding soil and revealed that the grid form improved method is effective in liquefaction mitigation [4] [5] [6].

Regarding the design procedure and guidelines of the grid type improvement method, the Ministry of Construction proposed that the grid wall spacing to thickness of loose sand layer ratio $(\mathrm{L} / \mathrm{H})$ should be smaller than about 0.8 to assure the effect of liquefaction prevention [7]. Therefore, the typical grid spacing ratio used as common design practice for construction site is about $0.5-0.8$. Nevertheless, it is still insufficient to reduce the risk completely because the effectiveness of improvement method in liquefaction prevention is depending on many factors, such as cement column stiffness, wall thickness and grid wall spacing to grid height ratio, etc. [8]. The research group of the Port and Airport Research Institute also indicated that the grid spacing is a key factor to control the generating of excess pore water pressure and settlement in liquefaction mitigation [9]. It means that the amount of excess pore water pressure and settlement could escalate with the increase in grid spacing ratio. Besides, jet grouting machines are huge in size and difficult to utilize in city areas. To arrive at the most economic and efficient design methodology, some researchers have been developed new design reinforced methods. For instance, Isobe et al. proposed a hexagonal grid form method, in which hexagonal shaped grid form with small diameter of jet grouted walls $(0.16 \mathrm{~m})$ were utilized for liquefaction countermeasure [10].

In this study, a new countermeasure method, the grid form with additional horizontal slabs, is introduced as shown in Figure 3. Herein, grid spacing ratio of 0.2 was considered, which is equivalent to $2 \mathrm{~m}$ and $10 \mathrm{~m}$ of grid wall spacing and thickness of the liquefiable layer, respectively. The grid wall $(0.15 \mathrm{~m}$ diameter) and horizontal slab ( $0.5 \mathrm{~m}$ thickness) are constructed by means of jet grouting procedure, in which a small machine is used for easy access to the urban 


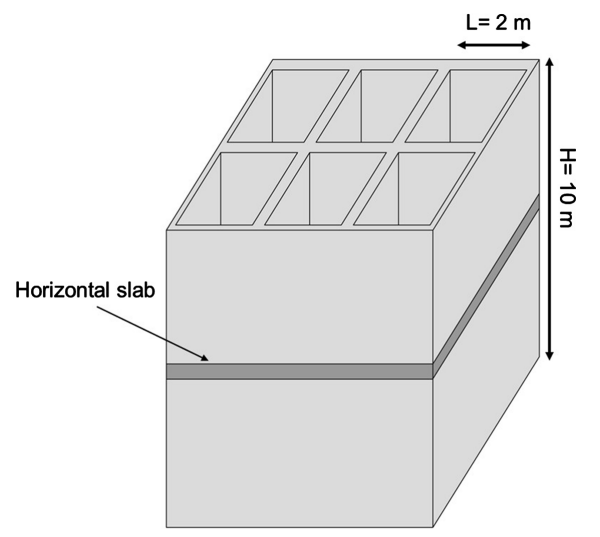

Figure 3. New grid type model for liquefaction mitigation.

areas and applicable to deploy near the boundaries of existing structures. In addition, the liquefiable loose soil is improved by the stiff jet grout grid wall in both vertical and horizontal directions, thereby controlling shear force generated in the ground during an earthquake. And also, this horizontal slab is expected to act as bracing element between the walls to increase the grid wall stability by reducing the shear forces and bending moments. In order to evaluate the effectiveness of grid form with the horizontal slab in reducing liquefaction risk, both physical modeling and numerical simulation were performed for unimproved case and improved case under different dynamic loading conditions. In experimental modelling for $1 \mathrm{~g}$ shaking table tests, the response of acceleration, excess pore water pressure ratio and settlement were used to assess the effectiveness of the soil improvement method. On the other hand, settlement and distribution of shear strain under the building were evaluated for unimproved ground and improved ground by using the numerical PLAXIS software.

\section{Shaking Table Test}

Shaking table test is one of the essential requirements in earthquake geotechnical engineering that helps in studying the behavior and performance of earth structures during earthquake. Several researches have been carried out to understand the liquefaction failure mechanisms and the effect of various ground improvement techniques by using shaking table tests. Koga and Matsuo performed a series of shaking table tests for embankment models on liquefiable sandy ground [11]. Most recently, Bahamanpour et al. also stated the use of $1 \mathrm{~g}$ shaking table tests in assessing the effect of underground columns on the mitigation of liquefaction [12]. According to the results, the shaking table test provides valuable insight into liquefaction, post-earthquake settlement, rate of generation and dissipation of excess pore water pressure and soil-structure response. In this research, the $1 \mathrm{~g}$ shaking table tests were conducted for unimproved case and improved case to verify the effectiveness of a new countermeasure method in liquefaction mitigation. Acceleration, excess pore water pressure and settlement were measured during the experiments. 


\subsection{Experimental Set up}

The experimental set up and instrumentations for the tests are shown in Figure 4. The test models were constructed in a transparent soil container made of acrylic plates and steel frames. The dimensions of the soil container were 1800 mm Length $(L), 400 \mathrm{~mm}$ Width $(W)$ and $800 \mathrm{~mm}$ Height $(H)$. In the experiments, a prototype to model ratio $(N)$ of 33 and the similitude relationship proposed by Iai [13] were adopted to determine the various parameters for the test. The ground in the model was prepared in two layers using Toyoura sand; a non-liquefiable base-layer with relative density, $D_{r} 90 \%$, and an upper liquefiable soil layer with relative density, $D_{r} 50 \%$. The physical properties of the Toyoura sand are shown in Table 1. For construction of ground model, the lower hard layer was prepared by heavy compaction to achieve the desired density while the upper liquefiable sand layer was prepared using air pluviation by controlling height and flow rate of sand pouring. In the improved case, the grid form model was placed at the center of the container. For model construction, the stiff grid wall was made of acrylic plate with the stiffness of $2000 \mathrm{MPa}$, and its thickness and length were determined according to the simulate law. The length of the grid wall was set as the same measurement with the thickness of upper loose sand layer, and thus, the bottom of the improved grid form model rested on top of the non-liquefiable layer.

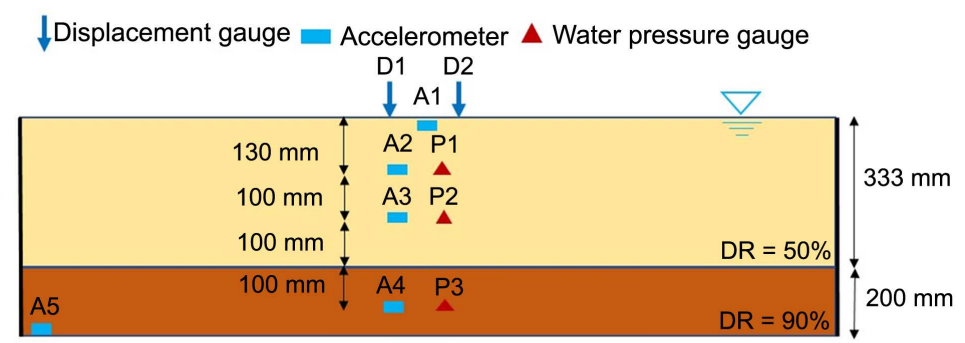

(a)

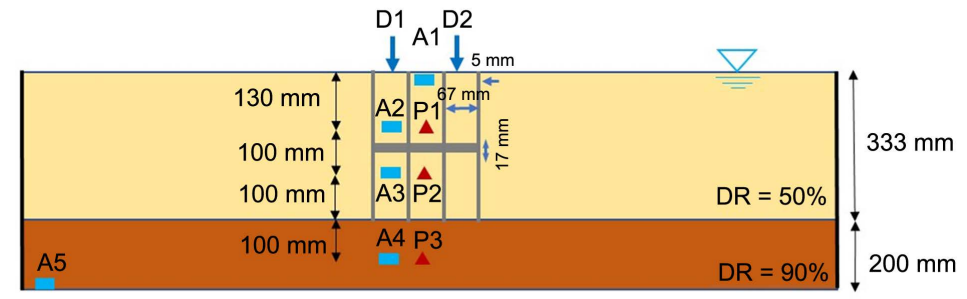

(b)

Figure 4. Experimental setup and instrumentations for $1 \mathrm{~g}$ tests in model scale (a) Unimproved case (b) Improved case.

Table 1. Physical properties of Toyoura sand.

\begin{tabular}{cccccc}
\hline $\begin{array}{c}\text { Soil particle } \\
\text { density, } \\
\left(\mathrm{g} / \mathrm{cm}^{3}\right)\end{array}$ & $\begin{array}{c}\text { Maximum } \\
\text { dry density, } \\
\left(\mathrm{g} / \mathrm{cm}^{3}\right)\end{array}$ & $\begin{array}{c}\text { Minimum } \\
\text { dry density, } \\
\left(\mathrm{g} / \mathrm{cm}^{3}\right)\end{array}$ & $\begin{array}{c}\text { Maximum } \\
\text { Void ratio }\end{array}$ & $\begin{array}{c}\text { Minimum } \\
\text { Void ratio }\end{array}$ & $\begin{array}{c}\text { Mean } \\
\text { grain size, } \\
(\mathrm{mm})\end{array}$ \\
\hline 2.64 & 1.645 & 1.335 & 0.973 & 0.609 & 0.17 \\
\hline
\end{tabular}


The test models were equipped with accelerometers, displacement gauges and pore water pressure gauges to measure horizontal accelerations along the shaking direction, settlement and excess pore water pressure during and after the seismic loadings. To minimize the boundary effects on the measurements, the sensors were installed at the center of the container. After the model construction, saturation of the model was carried out from the bottom of sand layer using steady water flow with very low discharge rate to dissolve air and to fully saturate the voids.

\subsection{Input Dynamic Loadings and Test Procedure}

The aim of the present research is to develop a new countermeasure method to mitigate building damage against liquefaction induced by moderate or large scaled earthquakes. Therefore, two types of seismic loadings were adopted. These input loadings were applied at the bottom of the soil container in the form of sinusoidal acceleration waves. The testing step, amplitude, frequency and time duration of seismic loadings applied in the experiments are presented in Table 2.

\section{Experimental Result and Discussion}

A set of $1 \mathrm{~g}$ shaking table tests for unimproved and improved conditions were performed under different loading circumstances. The purposes of the experiments were to determine 1) the effectiveness of new mitigation method against liquefaction 2) the seismic response of liquefiable soil with and without the presence of improved grid-form under dynamic conditions. The effectiveness of the jet-grout column with the horizontal slab was evaluated based on the acceleration, excess pore water pressure as well as settlement during and after the seismic loadings.

\subsection{Acceleration}

Liquefaction triggered the serious deterioration of soil shear strength and stiffness. As the shear modulus of the soil becomes significantly smaller, the shear wave energy hardly reaches to the soil surface, and acceleration attenuation behavior occurs at the ground surface. Hence, the acceleration response of the soil surface becomes one of the identifying factors in evaluating the performance of the soil improvement techniques. The soil acceleration histories at the soil surface together with the input motion $(0.3 \mathrm{~g})$ for the unimproved case and the improved case are presented in Figure 5. In the unimproved case, it can be seen

Table 2. Earthquake loading conditions.

\begin{tabular}{cccc}
\hline Step & Acceleration $(\mathrm{g})$ & Frequency $(\mathrm{Hz})$ & Time $(\mathrm{s})$ \\
\hline 1 & 0.2 & 2 & 10 \\
2 & 0.3 & 4 & 10 \\
\hline
\end{tabular}



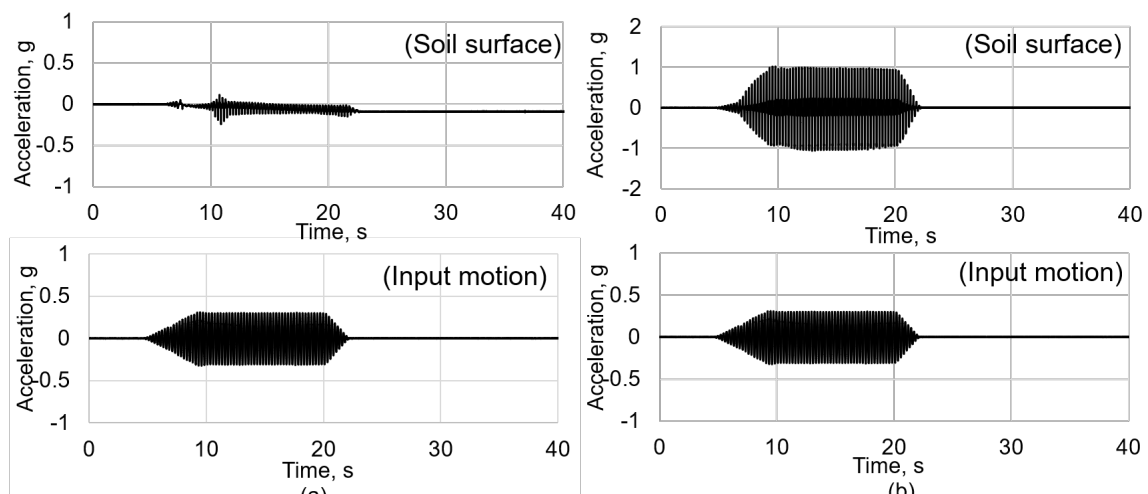

(a)

(b)

Figure 5. Time histories of acceleration under $0.3 \mathrm{~g}$ loading (a) unimproved case (b) improved cases.

that the surface acceleration was notably de-amplified as a result of soil softening induced by larger generation of excess pore water pressure, indicating complete liquefaction. Conversely, the soil stiffness remained sustaining within the grid form for the improved case. Thus, seismic wave was effectively transferred from the base to the soil surface within the grid form, resulting amplification behavior occurred at the soil surface. In view of this phenomenon, the new countermeasure method by means of grid form with the horizontal slab, indicates a potential to control the liquefaction occurrence against the earthquake.

\subsection{Excess Pore Water Pressure}

The grid spacing ratio is one of the essential design factors for the grid type improvement method. It is because, the stiffer grid wall provides shear reinforcement to the soil and minimize the excess pore water pressure generating against earthquake. Expressly, the excess pore water pressure ratio can increase with the grid spacing ratio due to insufficient reinforcement to the soil. Hence, the ratio of grid wall spacing to the improved grid height $(L / H)$ is used as 0.2 in this study. In aspect of the time histories of excess pore water pressure ratio $\left(\Delta u / \sigma_{v}^{\prime}\right)$ at different depths, the performances of the improved ground model under the different earthquake loadings are presented comparing to the unimproved ground model in Figure 6 and Figure 7. In these cases, the mitigation effect of the jet grout column with horizontal slab method was observed at pore water pressure gauge P1 and P2 since they were installed in the liquefiable loose sand layers. In unimproved case, the corresponding loose sand layer was in a complete liquefied state as the excess pore water pressure ratio $\left(\Delta u / \sigma_{v}^{\prime}\right)$ reached to lunder both $0.2 \mathrm{~g}$ and $0.3 \mathrm{~g}$ loadings. From the time histories of unimproved case, the chronological agreement between the maximum excess pore water pressure and the drastic attenuation of surface acceleration amplitude can be obviously seen in Figure 5 and Figure 7. In this case, the surface acceleration amplitudes attenuated as a result of high level of excess pore pressure generated in the soil during the $0.3 \mathrm{~g}$ cyclic loading. On the contrary, for the improved 


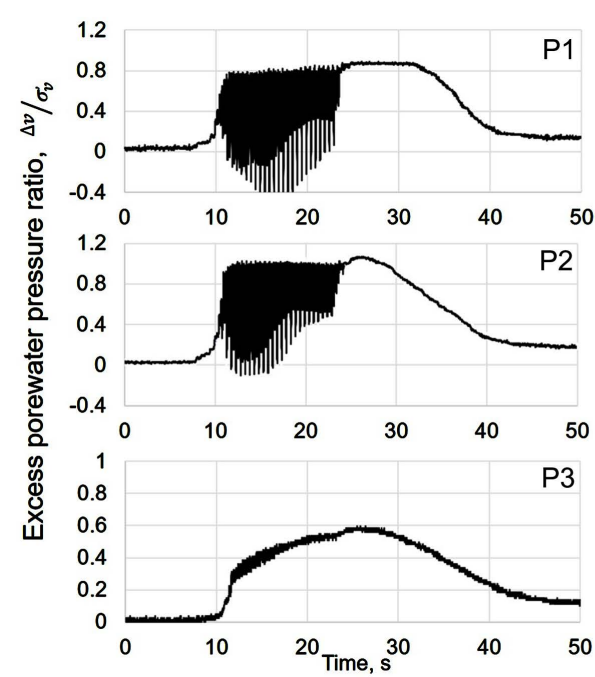

(a)
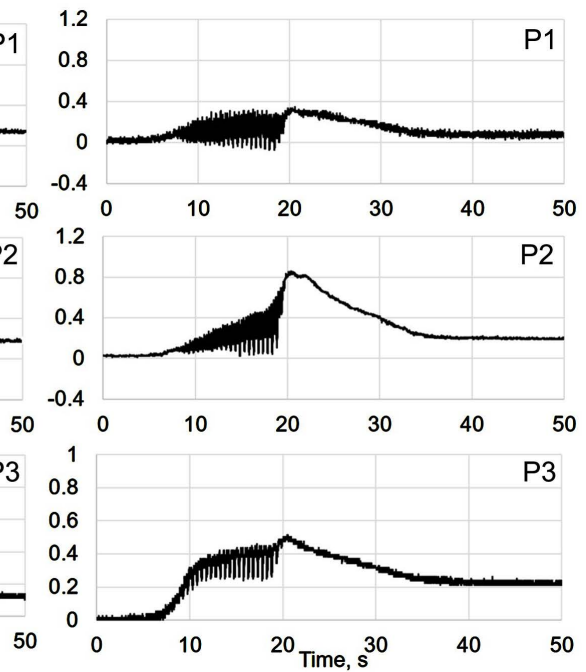

(b)

Figure 6. Time histories of excess pore water pressure ratio under $0.2 \mathrm{~g}$ loading (a) unimproved case (b) improved case.

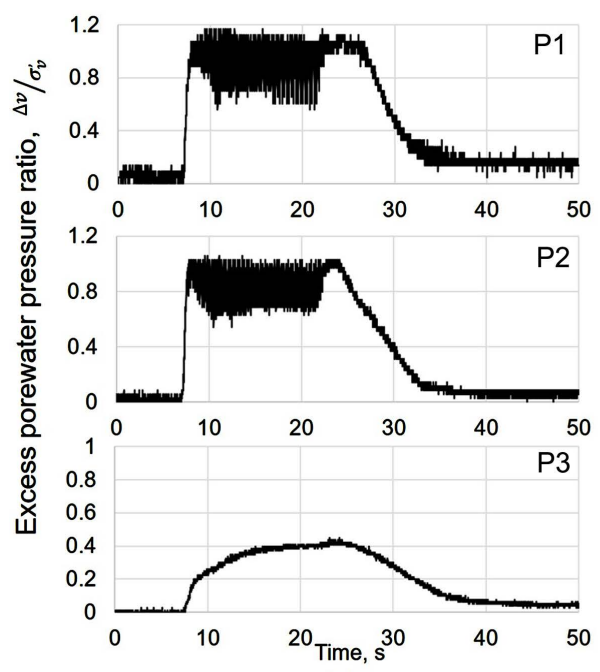

(a)

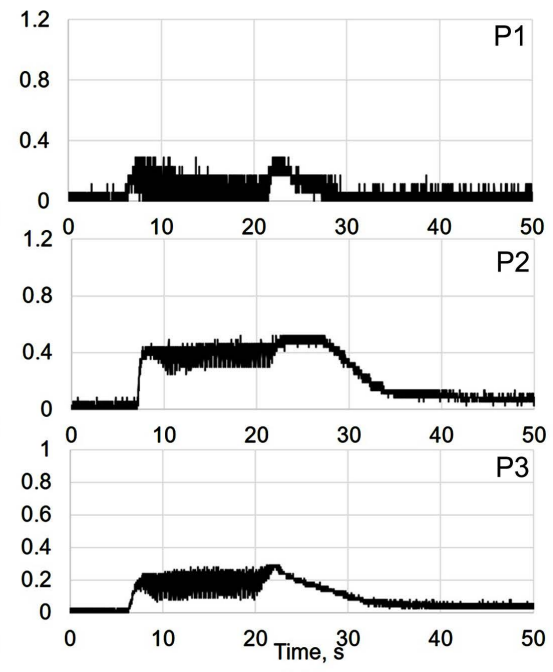

(b)

Figure 7. Time histories of excess pore water pressure ratio under $0.3 \mathrm{~g}$ loading (a) unimproved case (b) improved case.

case, the excess pore water pressure ratios measured at P1 and P2 in the soil within the grid form were found to be less than 1 , showing respective peak values about of 0.3 and 0.8 under $0.2 \mathrm{~g}$ loading and 0.3 and 0.5 under $0.3 \mathrm{~g}$ loading. Moreover, the dissipation duration of excess pore water pressure at post shaking in the unimproved case took a longer than that in the improved case. It indicates that the unimproved ground experienced substantial cyclic shear deformation and negative dilatancy was accumulated during the cyclic loading. At the pore water pressure gauge $\mathrm{P} 3$, the excess pore water pressure ratio did not reach to 1 as expected, since it was installed in the non-liquefiable hard layer. In view of the measurements at P1 and P2, it can be stated that the use of small spacing ratio of 
grid form with horizontal slab is effective in controlling the built up of excess pore water pressure.

\subsection{Settlement}

As aforementioned, the ground settlement is consequences of soil liquefaction that adversely harm buildings after an earthquake. Therefore, countermeasure against structure damages due to the ground subsidence is essential in liquefaction mitigation. To address this, the efficiency of the new mitigation method on ground settlement control was studied under the different dynamic loadings. In Figure 8, the settlement result of the unimproved case was compared with the settlement result measured within the grid form of the improved case. With reference to Figure 8 , in both cases, over $90 \%$ of the ground settlements occurred during shakings, while only a small amount of settlement was contributed by post-shaking soil reconsolidation owing to excess pore water pressure dissipation. Therefore, Liu and Dorby [14] mentioned that the inertial forces due to shaking plays a decisive role in the deformation. In grid form improved method, the stiffer and confined grid walls help to resist earthquake induced destructive forces, thereby reducing the seismic force in the native soil within the grid form. As a consequence, the settlement within the grid form remains small. Hence, the total settlement of unimproved case was found to be about $17 \mathrm{~mm}$ after the $0.2 \mathrm{~g}$ and $0.3 \mathrm{~g}$ shaking loadings, with a rapid initial settlement due to intensive soil softening. However, a delayed response of initial settlement and a lesser amount of total settlement value of about $5 \mathrm{~mm}$ were observed in the improved case. In addition, the larger amount of settlement occurred in the $0.2 \mathrm{~g}$ loading case compared to the $0.3 \mathrm{~g}$ in unimproved case. It indicates that the unimproved ground underwent large shear deformation imposed by the initial test loading of $0.2 \mathrm{~g}$, accompanying with larger amount of ground settlement. Subsequently, only less amount of settlement was observed in the unimproved case under $0.3 \mathrm{~g}$ loading because of soil densification caused by the previous loading. In the improved case, the small amount of settlement occurred under both $0.2 \mathrm{~g}$ and $0.3 \mathrm{~g}$ loadings since the stiff grid wall provides reinforcement and reduces destructive forces induced shaking. In view of the abovementioned observations, it can be determined that the ground settlement could be remarkably reduced by using the proposed jet-grout column with horizontal slab reinforcing technique.

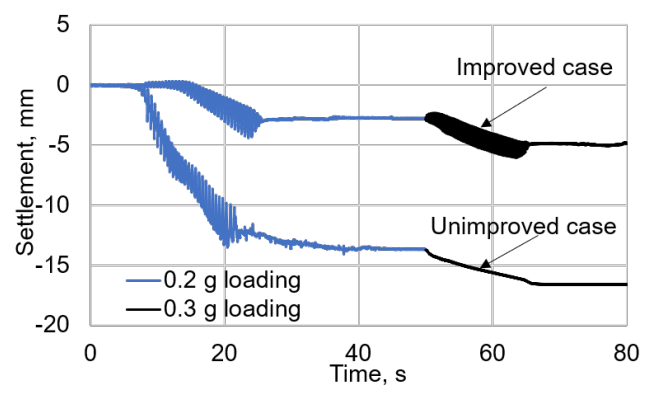

Figure 8. Time histories of settlement under $0.2 \mathrm{~g}$ and $0.3 \mathrm{~g}$ loadings. 


\section{Numerical Simulation}

A partial or total structure collapse may result in liquefaction -induced settlements under a structure, especially when differential settlement takes place. In order to consider remediation measures on vulnerable housing in liquefaction prone regions, numerous researches have been developed under the topic of soil liquefaction and ground improvement. The researches covered both experimental and numerical approaches to predict the soil and structural behavior under the earthquake condition [15] [16] [17]. Dashti and Bray [18] studied the mechanism of volumetric and deviatoric settlement components in shallow foundation settlement induced by liquefaction, using UBCSAND constitutive soil model validated with centrifuge tests. The experimental and numerical results reveal that the building settlements accumulate mainly during the shaking period and the deformation are especially associated with deviatoric strains attribution as the result of foundation contact pressure and the response of soil and structure inertial force. Volumetric strain is accounted only for a small portion of the accumulated seismic settlement since drained condition is not allowed at this stage. Therefore, the deviatoric strains from the shear loss in the soil is one of the pivotal mechanisms driving the building settlement. In this section, a set of numerical studies were conducted using PLAXIS software to assess the effectiveness of jet-grout wall with horizontal slab in building settlement mitigation against liquefaction. The UBC3D-PLM model was used to capture the liquefiable soil behavior and deviatoric displacements induced with the presence of the building under the earthquake loading. The effectiveness of new countermeasure method is evaluated based on the settlement due to distribution of deviatoric strains during the earthquake.

\subsection{Numerical Model and Boundary Conditions}

The numerical studies aim to provide insight into the seismic performance of jet grouted wall in liquefiable soil under the building. Hence, the numerical simulations for building on unimproved and improved ground conditions were studied. In terms of soil profile for numerical model, an idealized three-layer of soil column was used. The water table was assumed to be located at a depth of $1 \mathrm{~m}$ under the ground level. The effectiveness of ground improvement was measured at the 10 -m-thick sand layer with $D_{r} 50 \%$, which is overlying $16 \mathrm{~m}$ of clay. The underlying stratum of sand and clay layers was presumed to be bedrock where the earthquake data of the Kumamoto (2016) was imposed with the maximum peak ground acceleration of $0.24 \mathrm{~g}$. This earthquake data was recorded at the Mashiki site where major building damage occurred due to the strong motion. The time history of acceleration was depicted in Figure 9. In the simulation, a two-story building with $7 \mathrm{~m}$ height and $10 \mathrm{~m}$ width, was modelled on the liquefiable soil. As a reference model, the numerical model considered for improved case in the analyses is shown in Figure 10. All units used in the numerical model are in prototype scale. 


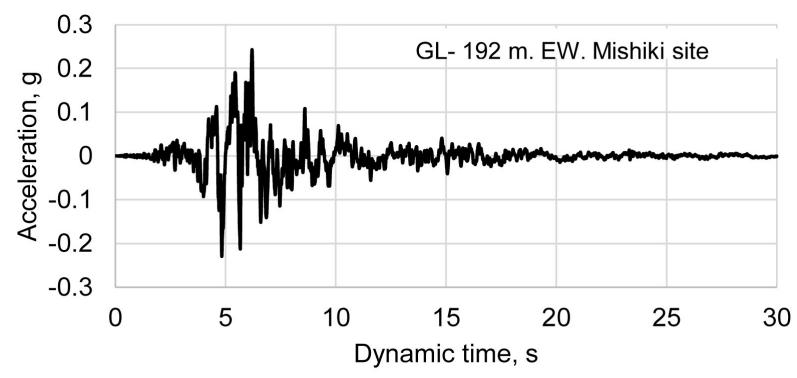

Figure 9.Seismic input motion applied in the Analyses

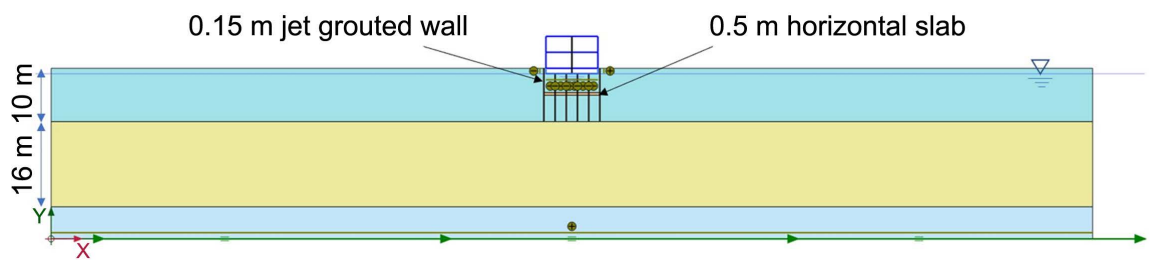

Figure 10. Finite element model of the improved case.

In these numerical analyses, 15 nodes triangular plain-strain elements were used to create a mesh distribution. Fine mesh option was selected in the analyses to meet the minimum required finite element length as suggested by Kuhlemyer and Lysmer [19]. Moreover, the mesh closer to the region of the embedded footing was refined to provide a better discretization in the area of interest. The horizontal dimension of the soil profile was chosen to be large enough to minimize the boundary condition effect. Default fixities were applied for the static stages. In the dynamic phase, the vertical boundaries were modelled with tied degree of freedom while reflected base was selected at the base. To define the Rayleigh damping coefficients, damping ratios and related frequencies were considered based on the proposed method by Hudson et al. [20] .

\subsection{Constitutive Models and Parameter Determination}

In this study, an elasto-plasticity based model, UBC3D-PLM was used to measure the development of excess pore water pressure and capture the onset of liquefaction in loose sand under the earthquake loading. The liquefaction UBC3D-PLM model is a 3D generalized extension of the UBCSAND model introduced by Puebla et al. [21]. Tsegaye [22] developed the first version of this generalized 3D formulation of the model and then, Petalas and Galavi [23] further improved the model capabilities, especially for monotonic loading. In the model, the primary and secondary yield surfaces are utilized to account for the effect of soil densification and to predict the smooth transition into the liquefaction state under undrained loading. Additionally, a soil densification rule, $f_{\text {dens }}$ is implemented to better predict the evolution of pore pressures during cyclic loading.

Even though UBC3D-PLM is an advanced model, it is relatively simple to apply due to its reasonable number of parameters that can be extracted from la- 
boratory or in-situ tests. The input liquefaction parameters are derived based on the corrected clean sand equivalent SPT blow-count number $\left(N_{1}\right)_{60}$ in accordance with the proposed correlations by Beaty and Byrne [24]. However, the selection and determination of parameters play a significant role to obtain reliable results. In this study, the constitutive parameters were calibrated based on the curve fitting of the cyclic resistance ratios of sand with different relative densities, reproduced by means of cyclic direct simple shear test implemented in soil test facility of PLAXIS. The undrained cyclic direct shear tests were performed under stress-controlled conditions by applying a constant shear stress amplitude in each step for a total of 20 cycles for different relative densities. To determine the CRR of the soil using the UBC3D-PLM model, the criterion for the beginning of the liquefaction process introduced by Seed et al. [25] was used. For a seismic magnitude of 7.5, liquefaction is found to occur when $r_{u}$ reached greater than 0.85 for 15 load cycles. Figure 11(a) presents the development of excess pore water pressure ratio against number of cycles for different relative densities. Figure 11(b) shows the comparison of cyclic strength curves obtained from the numerical and the semi-empirical methods.

The results show that the PLAXIS UBC3D-PLM model is in good agreement with other cyclic strength curves. The properties of soil parameters used in UBC3D-PLM model are shown in Table 3. However, the UBC3D-PLM model is
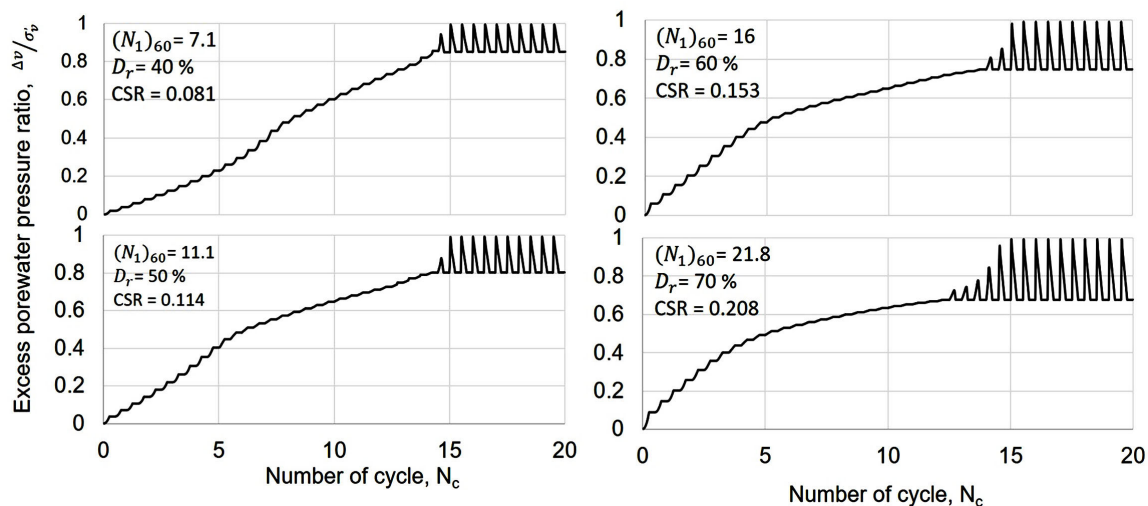

(a)

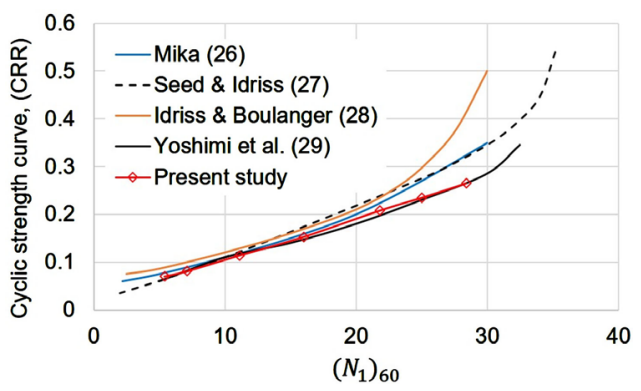

(b)

Figure 11. Calibration for parameters used in UBC3D-PLM model (a) development of pore water pressure ratios with number of cycles for different relative densities (b) cyclic strength curve obtained from UBC3D-PLM in comparison with other curves. 
Table 3. Parameters used in UBC3D-PLM model.

\begin{tabular}{ccc}
\hline Parameter & Symbol & Value \\
\hline Peak friction angle $\left(^{\circ}\right)$ & $\varphi_{p}^{\prime}$ & 33 \\
Constant volume friction angle $\left(^{\circ}\right)$ & $\varphi_{c v}^{\prime}$ & 32.1 \\
Elastic shear modulus number & $K_{G}^{e}$ & 967.67 \\
Elastic bulk modulus number & $K_{B}^{e}$ & 677.37 \\
Plastic shear modulus number & $K_{G}^{P}$ & 458.4 \\
Elastic shear modulus index & $m e$ & 0.5 \\
Elastic bulk modulus index & $n e$ & 0.5 \\
Plastic shear modulus index & $n p$ & 0.4 \\
Failure ratio & $R_{f}$ & 0.77 \\
Densification factor & $f_{\text {dens }}$ & 0.45 \\
Post liquefaction factor & $f_{\text {post }}$ & 0.02 \\
Corrected standard penetration test & $\left(N_{1}\right)_{60}$ & 11.1 \\
\hline
\end{tabular}

not appropriate to use in static analysis since the parameters used in the model are designated to evaluate liquefaction in loose soils and suitable only for dynamic calculation [30]. Therefore, Hardening soil model was used in initial static phase to correctly generate the stress state of liquefiable soil prior to the dynamic phase. Furthermore, Hardening soil model was also applied in clay layer to simulate the behavior of the stress dependent stiffness and cyclic subjected by earthquake loading. The properties of soil parameters used in Hardening soil model areee tabulated in Table 4 . The underlying bed rock was modelled as Linear Elastic model with unit weight $\left(22 \mathrm{KN} / \mathrm{m}^{3}\right)$, Young modulus (6000 MPa) and Poisson's ratio (0.2). In the improved case, Mohr-Coulomb model was employed in jet grout columns modelling. Elasticity modulus, Poisson's ratio and Undrained shear strength of the columns were selected as $1000 \mathrm{MPa}, 0.2$ and 1 $\mathrm{MPa}$, respectively. For the building structure, a simplified modelling approach was adopted. In this approach, linear elastic material is used for the structural elements. The walls and floors were modelled with plate element while more rigid elements were applied for the foundation slab. And, the columns were modelled as node to node anchors in $3 \mathrm{~m}$ spacing.

\subsection{Numerical Results and Discussions}

The contour of after earthquake vertical displacement for the unimproved and the improved cases are depicted in Figure 12. In the unimproved case, the considerable punching failure of the building occurred due to the strong ground shaking. As a result, large settlement and uplift of the ground were observed underneath and surrounding of the building, respectively. The maximum vertical soil displacement observed below the bottom left of the structure because the 


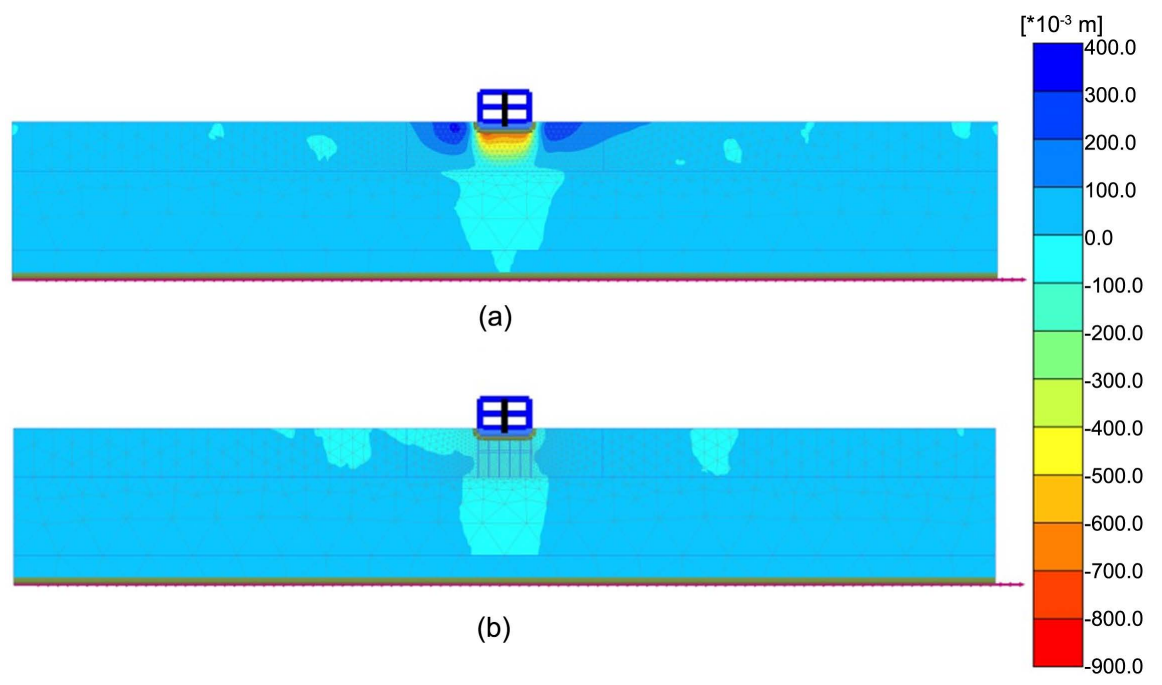

Figure 12. Vertical displacements at the end of the earthquake (a) unimproved case (b) improved case.

Table 4. Parameters used in Hardening soil model.

\begin{tabular}{cccc}
\hline Parameter & Symbol & Sand & Clay \\
\hline Unit weight, $\left(\mathrm{KN} / \mathrm{m}^{3}\right)$ & $\gamma_{\text {sat }}$ & 20 & 16 \\
Effective cohesion, $\left(\mathrm{KN} / \mathrm{m}^{2}\right)$ & $c^{\prime}$ & 0 & 22 \\
Effective friction angle $\left(^{\circ}\right)$ & $\varphi^{\prime}$ & 33 & 13 \\
Dilatancy angle $\left(^{\circ}\right)$ & $\psi$ & 1 & - \\
Secant Modulus, $\left(\mathrm{KN} / \mathrm{m}^{2}\right)$ & $E_{50}^{\text {ref }}$ & 20,380 & 5435 \\
Tangent stiffness for primary oedometer loading, $\left(\mathrm{KN} / \mathrm{m}^{2}\right)$ & $E_{\text {oed }}^{\text {ref }}$ & 20,380 & 5436 \\
Unloading/reloading stiffness $\left(\mathrm{KN} / \mathrm{m}^{2}\right)$ & $E_{\text {ur }}^{\text {ref }}$ & 61,130 & 16,310 \\
Power of stress-level decency & $m$ & 0.5 & 0.8 \\
\hline
\end{tabular}

structure rotated about its bottom right-hand edge. And, this rotating mechanism caused the significant soil heaving at the other side of the structure. On the other hand, such ground failure mechanism was not encountered in the improved case. The measured settlements underneath the building were compared between unimproved case and improved cases, as shown in Figure 13. The reinforced ground with new grid form shows considerably lesser settlement compared to the unreinforced ground. The net accumulated settlement at the end of the earthquake in unimproved and improved cases were found to be about $760 \mathrm{~mm}$ and $80 \mathrm{~mm}$, respectively. It indicates that the stiffer jet-grouting wall can help to restrict the soil displacement under the foundation and able to provide bearing support to vertical load imposed by structure. Moreover, lateral flow of foundation soil is one of the major causes resulting in ground settlement during the earthquake. In unimproved case, the soil particles mainly under the building can freely move, which resulting in large deformation. However, the 
horizontal or lateral movement of the soil particles were restrained by the grouted wall in improved case as distinctly shown in Figure 14. Therefore, only small settlement was observed in improved ground.

Furthermore, the deviatoric displacement mechanism, which governing the response of the building located on the liquefiable soil with and without improvement, were investigated to confirm the effect of the proposed liquefaction countermeasure method in settlement control. Figure 15 shows the distribution of the total deviatoric strain underneath the building at the end of the earthquake in unimproved and improved cases. As an obvious observation, the maximum values of total deviatoric strain accumulations occurred underneath the corner of the building at the end of earthquake in the unimproved case. It might be the consequence of the soil-structure interaction induced by foundation rocking during the earthquake, prior to a punching-type failure mechanism. In addition, the magnitude of horizontal displacement was large in these locations. On the contrary, there was only negligible amount of deviatoric strain distribution observed in the improved case. This phenomenon took place because the structure only experienced small amount of settlement against the earthquake. In view of the above-mentioned numerical results, the proposed jet grout column with horizontal slab method considerably increases the serviceability of the foundation by means of reinforcing to the loose sand and increasing the strain characteristic, and effective in mitigating ground damage.

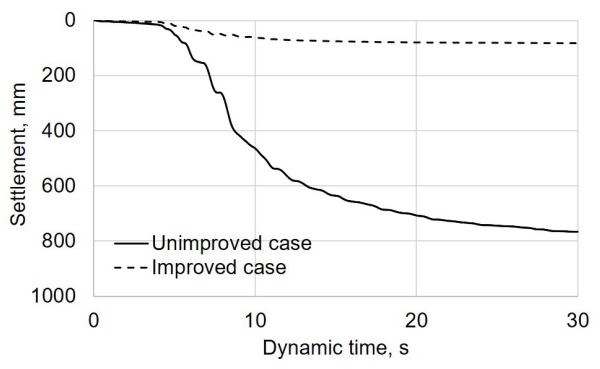

Figure 13. Time histories of settlement obtained from numerical analyses.

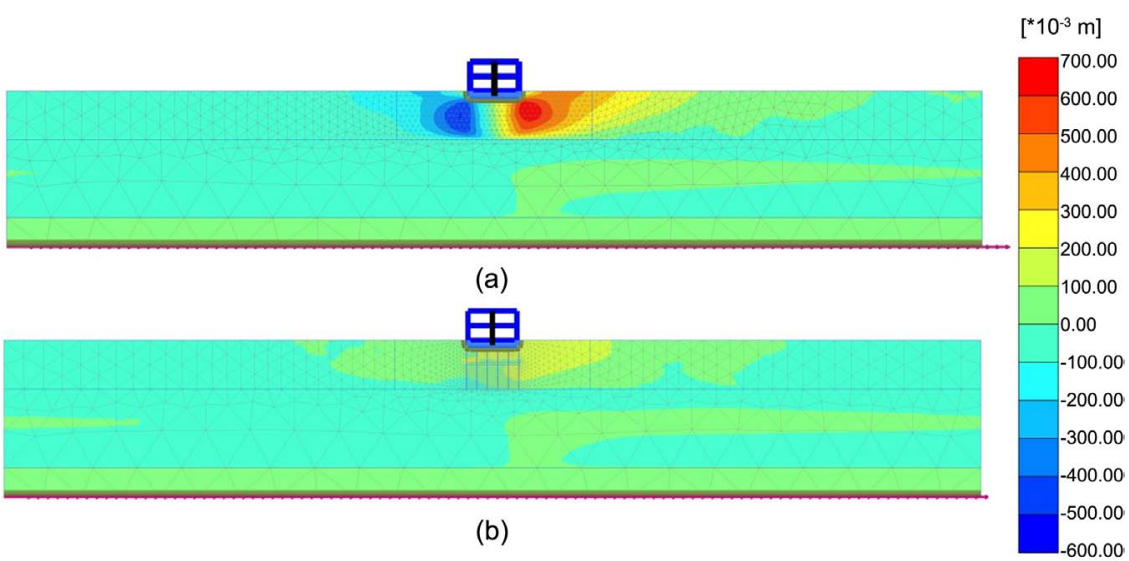

Figure 14. Horizontal displacement at the end of the earthquake (a) unimproved case (b) improved case. 


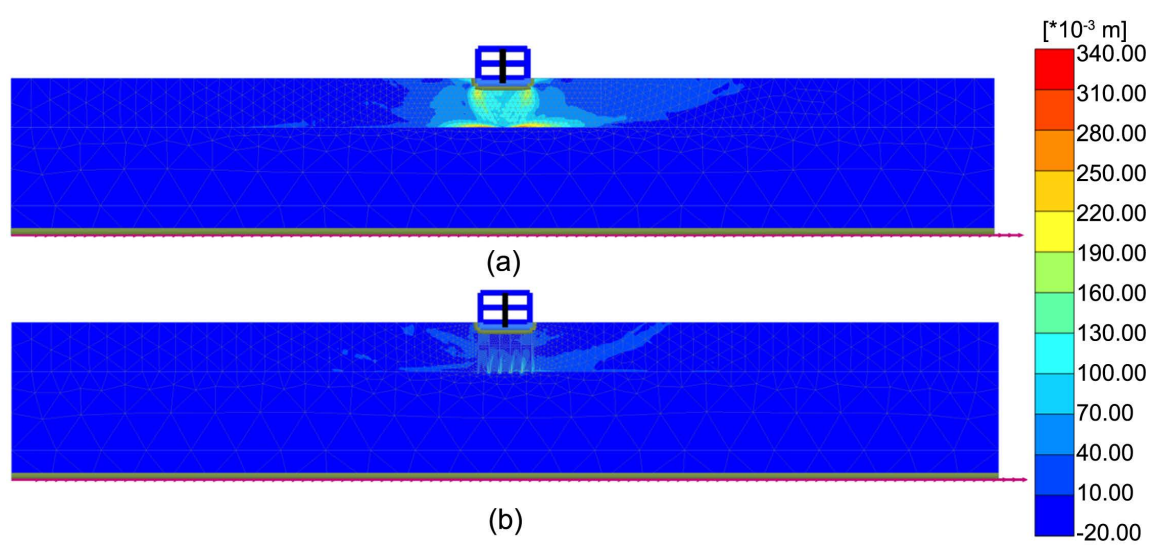

Figure 15. Distribution of the deviatoric shear strains (a) unimproved case (b) improved case.

\section{Conclusions}

Mitigation techniques have become essential for buildings in vulnerable areas which are prone to severe damage caused by earthquake induced liquefaction. In this study, a new countermeasure technique of grid form with horizontal slab was introduced and its effectiveness in liquefaction mitigation was assessed by experimental modeling and numerical modeling. The following conclusions could be derived based on the experimental results and numerical results.

1) Amplification behavior of the surface acceleration observed in the improved case, clearly indicates that the shear strength degradation of the soil within the grid form was effectively sustained.

2) The relatively small built up of excess pore water pressure in the soil within the grid form compared to the unimproved case shows that the use of grid spacing ratio 0.2 in a new improved method is effective in liquefaction mitigation.

3) The ground settlement is one of the pivotal measurements to evaluate the effect of ground improvement techniques. Considering both experimental and numerical results, the new grid form with horizontal slab improved method is significantly effective in settlement control. The numerical results show that the jet grout walls are able to increase bearing support for shallow foundations and maintain the structural integrity against liquefaction.

4) The finite element analysis helped in better understanding of ground displacement as well as shear strain distribution of the foundation soil underneath the existing building, for both unimproved case and improved case. It clearly depicts that the large settlement in the unimproved case was associated with the horizontal movements of foundation soils. On the other hand, the jet grout walls in the improved case helped to restrain soil movement and improve the strain characteristic, resulting lesser lateral displacement.

In summary, installation of the jet grout grid form with horizontal slab under the building, helps to provide considerably improvement in the serviceability of the foundation, protect the influence of the seismic effects on the soils and re- 
duce the liquefaction potential of the loose soil. Nevertheless, in order to achieve more useful and efficient design for the grid type method, it is required to perform further detailed studies such as the external and internal stability of the grid wall, and the mechanism of the horizontal slab enhancement in ground improvement.

\section{Acknowledgements}

The first author would like to gratefully acknowledge to MEXT scholarship program for financially supporting for study in Japan.

\section{Conflicts of Interest}

The authors declare no conflicts of interest regarding the publication of this paper.

\section{References}

[1] Hazarika, H., Kokusho, T., Kayen, R.E., Dashti, S., Fukuoka, H., Ishizawa, T., Kochi, Y., Matsumoto, D., Hirose, T., Furuichi, H., Fujishiro, T., Okamoto, K., Tajiri, M. and Fukuda, M. (2018) Geotechnical Damage Due to the 2016 Kumamoto Earthquake and Future Challenges. Lowland Technology International, 19, 189-204.

[2] Wakamatsu, K., Senna, S. and Ozawa K. (2017) Liquefaction during the Kumamoto Earthquakes on April 14 and 16, 2016. Proceedings of the International Workshop on the 2016 Kumamoto Earthquake, Fukuoka, Japan, 6 March 2017, 123-128.

[3] Suzuki, Y., Saitoh, S., Onimaru, S., Kimura, G., Uchida, A. and Okumura, R. (1996) Grid-Shaped Stabilized Ground Improved by Deep Cement Mixing Method against Soil Liquefaction for a Building Foundation. Tsuchi-to-Kiso, 44, 46-48. (in Japanese)

[4] Kitazume, M. (2016) Applications of Centrifuge Modelling to Liquefaction Mitigation Techniques. Proc. of Eurofuge, IFSTTAR, 1-3 June 2016, 1-18.

[5] Suzuki, K., Babasaki, R. and Suzuki, Y. (1991) Centrifuge Tests on Liquefaction-Proof Foundation. Proc. of the Centrifuge 91', Colorado, 13-14 June 1991, 1461-1464.

[6] Babasaki, R., Suzuki, K. and Suzuki, Y. (1992) Centrifuge Tests on Improved Ground for Liquefaction. Proceedings of the 10 th World Conference on Earthquake Engineering, Madrid, 19-24 July 1992, 1461-1464.

[7] Ministry of Construction (1999) Design and Construction Manual of Liquefaction Prevention Techniques (Draft). Joint Research Report, Japan, 186. (in Japanese)

[8] Namikawa, T., Koseki, J. and Suzuki, Y. (2007) Finite Element Analysis of Lattice-Shaped Ground Improvement by Cement-Mixing for Liquefaction Mitigation. Soils and Foundations, 47, 559-576. https://doi.org/10.3208/sandf.47.559

[9] Takahashi, H., Kitazume, M. and Ishibashi, S. (2006) Effect of Deep Mixing Wall Spacing on Liquefaction Mitigation. Proceedings International Conference on Physical Modelling in Geotechnics, Hong Kong, 4-6 August 2006, 585-590.

[10] Isobe, Y., Sekino, M., Takeuchi, J., Oonishi, T. and Nakajima, M. (2019) Numerical Analysis of Liquefaction Countermeasure Method by Hexagonal Grid Form Soil Improvement Using Jet Grouting Method. Proceedings of the 7 th International Conference on Earthquake Geotechnical Engineering, 7th ICEGE, Rome, 17-20 
June 2019, 3054-3061.

[11] Koga, Y. and Matsuo, O. (1990) Shaking Table Tests of Embankments Resting on liquefiable Sandy Ground. Soils and Foundations, 30, 162-174.

https://doi.org/10.3208/sandf1972.30.4 162

[12] Bahmanpour, A., Towhata, I., Sakr, M., Mahmoud, M., Yamamoto, Y. and Yamada, S. (2019) The Effect of Underground Columns on the Mitigation of Liquefaction in Shaking Table Test Model Experiments. Soil Dynamic and Earthquake Engineering, 116, 15-30. https://doi.org/10.1016/j.soildyn.2018.09.022

[13] Iai, S. (1989) Similitude for Shaking Table Test on Soil-Structure-Fluid Model in $1 \mathrm{~g}$ Gravitational Field. Soil and Foundations, 29, 105-118. https://doi.org/10.3208/sandf1972.29.105

[14] Liu, L. and Dobry, R. (1997) Seismic Response of Shallow Foundation on Liquefaction Sand. Journal of Geotechnical and Geoenvironmental Engineering, 123, 557-567. https://doi.org/10.1061/(ASCE)1090-0241(1997)123:6(557)

[15] Yoshimi, Y. and Tokimatsu, K. (1977) Settlement Buildings on Saturated Sand during Earthquakes. Soil and Foundations, 17, 23-38. https://doi.org/10.3208/sandf1972.17.23

[16] Towhata, I. and Rasouli, R. (2013) Attempts to Protect Personal Houses from Seismic Liquefaction Problem. Proceedings of the Seminar on Forensic Geotechnical Engineering, Master Concrete Resurfacing, 10-12 January 2013, 191-209.

[17] Yasuda, S., Sasaki, S., Noguchi, C. and Ozawa, N. (2013) Shaking Table Tests to Study the Possibility to Prevent Liquefaction-Induced Damage of Houses by Installing Sheet-Piles. Proceedings of 48 th Conference of Japanese Geotechnical Society, Toyama, 23-25 July 2013, 1759-1762.

[18] Dashti, S. and Bray, J.D. (2013) Numerical Simulation of Building Response on Liquefiable Soil. Journal of Geotechnical and Geoenvironmental Engineering, 139, 1235-1249. https://doi.org/10.1061/(ASCE)GT.1943-5606.0000853

[19] Kulemeyer, R. and Lysmer, J. (1973) Finite Element Method Accuracy for Wave Propagation Problems. Journal of Soil Mechanics and Foundation Division, 99, 421-427.

[20] Hudson, M., Idriss, I. and Beirkae, M. (1994) QUAD4M User'S Manual.

[21] Puebla, H., Byrne, P. M. and Phillips, R. (1997) Analysis of CANLEX Liquefaction Embankments: Prototype and Centrifuge Models. Canadian Geotechnical Journal, 34, 641-657. https://doi.org/10.1139/t97-034

[22] Tsegaye, A. (2010) Plaxis Liquefaction Model. External Report, PLAXIS Knowledge Base.

[23] Petalas, A. and Galavi, V. (2013) Plaxis Liquefaction Model Ubc3d-Plm. PLAXIS Knowledge Base.

[24] Beaty, M.H. and Byrne, P.M. (2011) Ubcsand Constitutive Model. Itasca UDM Website 904aR.

[25] Seed, H.B., Tokimatsu, K., Harder, L. F., Chung, M. and Riley, M. (1985) Influence of SPT Procedures in Soil Liquefaction Resistance Evaluations. Journal of Geotechnical Engineering, 12, 1425-1445. https://doi.org/10.1061/(ASCE)0733-9410(1985)111:12(1425)

[26] Makra, A. (2013) Evaluation of the UBC3D-PLM Constitutive Model for Perdition of Earthquake Induced Liquefaction on Embankment Dams. Delft University of Technology, Nederlands. 
[27] Seed, H.B. and Idriss, I.M. (1982) Ground Motions and Soil Liquefaction during Earthquakes. Earthquake Engineering Research Institute, Oakland.

[28] Idriss, I.M. and Boulanger, R.W. (2004) Semi-Empirical Procedures for Evaluating Liquefaction Potential during Earthquakes. Proceedings of the 11th International Conference on Soil Dynamics and Earthquake Engineering, and 3rd International Conference on Earthquake Geotechnical Engineering, Berkely, 7-9 January 2004, 32-56.

[29] Yoshimi, Y., Tokimatsu, K. and Ohara, J. (1994) In-Situ Liquefaction Resistance of Clean Sands over a Wide Density Range. Geotechnique, 44, 479-494. https://doi.org/10.1680/geot.1994.44.3.479

[30] PLAXIS 2D (2019) PLAXIS Material Models CONNECT Edition V20. 